\title{
The Maximum Size of an Edge Cut and Graph Homomorphisms
}

\author{
Suohai Fan ${ }^{1}$, Hongjian Lai ${ }^{2}$, Ju Zhou ${ }^{3}$ \\ ${ }^{1}$ Department of Mathematics, JiNan University, Guangzhou, China \\ ${ }^{2}$ Department of Mathematics, West Virginia University, Morgantown, USA \\ ${ }^{3}$ Department of Mathematics, Kutztown University, Kutztown, USA \\ E-mail: zhou@kutztown.edu \\ Received November 2, 2010; revised July 5, 2011; accepted July 12, 2011
}

\section{Abstract}

For a graph $G$, let $b(G)=\max \{|D|: D$ is an edge cut of $G\}$. For graphs $G$ and $H$, a map $\varphi: V(G) \rightarrow V(H)$ is a graph homomorphism if for each $e=u v \in E(G), \varphi(u) \varphi(v) \in E(H)$. In 1979, Erdös proved by probabilistic methods that for $p \geq 2$ with

$$
f(p)= \begin{cases}\frac{1}{2}+\frac{1}{2 p-2} & \text { if } p \equiv 0(\bmod 2), \\ \frac{1}{2}+\frac{1}{2 p} & \text { if } p \equiv 1(\bmod 2),\end{cases}
$$

if there is a graph homomorphism from $G$ onto $K_{p}$, then $b(G) \geq f(p)|E(G)|$. In this paper, we obtained the best possible lower bounds of $b(G)$ for graphs $G$ with a graph homomorphism onto a Kneser graph or a circulant graph and we characterized the graphs $G$ reaching the lower bounds when $G$ is an edge maximal graph with a graph homomorphism onto a complete graph, or onto an odd cycle.

Keywords: Maximum Edge Cuts, Graph Homomorphisms

\section{Introduction}

In this paper, the graphs we consider are finite, simple and connected. Undefined notation and terminology will follow those in [1]. Let $G$ be a graph. For disjoint nonempty subsets $X, Y \subseteq V(G)$, let $[X, Y]$ denote the set of edges of $G$ with one end in $X$ and the other end in $Y$. For a subset $S \subseteq V(G)$, let $\bar{S}=V(G) \backslash S$. An edge cut of $G$ is an edge subset of the form $[S, \bar{S}]$ for some nonempty proper subset $S \subseteq V(G)$. Define $b(G)=\max \{|D|: D$ is an edge cut of $G\}$.

For graphs $G$ and $H$, a map $\varphi: V(G) \rightarrow V(H)$ is a graph homomorphism if for each $e=u v \in E(G)$, $\varphi(u) \varphi(v) \in E(H)$. If there is a graph homomorphism from $G$ to $H$, then $G$ is called $H$-colorable. Suppose that $G$ is $H$-colorable. Then every graph homomorphism $\varphi: V(G) \rightarrow V(H)$ also induces a map $\varphi_{e}: E(G) \rightarrow E(H)$. If for any graph homomorphism $\varphi: V(G) \rightarrow V(H)$, and for any $e_{1}, e_{2} \in E(H)$, we always have $\left|\varphi_{e}^{-1}\left(e_{1}\right)\right|=\left|\varphi_{e}^{-1}\left(e_{2}\right)\right|$, then $G$ is called edge-uniformly $H$-colorable.

Throughout this paper, we use $K_{p}$ to denote the complete graph with $p$ vertices and $C_{2 p+1}$ to denote an odd cycle with $V\left(C_{2 p+1}\right)=\left\{v_{i}, i \in Z_{2 p+1}\right\}$ and $N\left(v_{i}\right)=\left\{v_{i-1}, v_{i+1}\right\}$.

Theorem 1.1 (Erdös [2]) Let $p \geq 2$ be an integer and

$$
f(p)=\left\{\begin{array}{cc}
\frac{1}{2}+\frac{1}{2 p-2} & \text { if } p \equiv 0(\bmod 2), \\
\frac{1}{2}+\frac{1}{2 p} & \text { if } p \equiv 1(\bmod 2) .
\end{array}\right.
$$

If there is a graph homomorphism from $G$ onto $K_{p}$, then $b(G) \geq f(p) \mid E(G)$.

Let $p$ and $q$ be two positive integers with $p \geq 2 q$. The Kneser graph $K_{p: q}$ is the graph whose vertices repre- 
sent the $q$-subsets of $\{0,1, \cdots, p-1\}$, where two vertices are connected if and only if they correspond to disjoint subsets.

Theorem 1.2 Let $p, q$ be two positive integers with $p \geq 2 q$. If there is a graph homomorphism from $G$ onto $K_{p: q}$, then $b(G) \geq \frac{2 q}{p}|E(G)|$.

Let $p$ and $q$ be two positive integers with $p \geq 2 q$. The circulant graph $K_{p / q}$ is the graph with vertex set $V(G)=\{0,1,2, \cdots, p-1\}$ and the neighbors of vertex $v$ are $\{v+q, v+q+1, \cdots, v+p-q\}$.

Theorem 1.3 Let $p, q$ be two positive integers with $p \geq 2 q$. If there is a graph homomorphism from $G$ onto $K_{p / q}$, then $b(G) \geq \frac{2 q}{p}|E(G)|$.

Theorem 1.4 Let $p, q$ be two positive integers with $p \geq 2 q$ and let

$$
f(p, q)= \begin{cases}\frac{p^{2}-4 q^{2}+4 q}{2 p(p-2 q+1)} & \text { if } p \text { is even, } \\ \frac{p^{2}-4 q^{2}+4 q-1}{2 p(p-2 q+1)} & \text { if } p \text { is odd. }\end{cases}
$$

If $G$ is an edge-uniformly $K_{p / q}$-colorable, then $b(G) \geq f(p, q) \mid E(G)$.

A graph $G$ is edge-maximal H-colorable if $G$ is $H$-colorable but for any graph $G^{\prime}$ such that $G^{\prime}$ contains $G$ as a spanning subgraph with $\left|E\left(G^{\prime}\right)\right|>|E(G)|$, $G$ is not $H$-colorable.

In Section 2, we prove an associate Theorem which will be used in the proofs of other theorems. In Subsection 3.1, we give an alternative proof of Theorem 1.1 and characterize the graphs $G$ reaching the lower bound in Theorem 1.1 when $G$ is edge-maximal $K_{p}$-colorable. In Subsection 3.2, we show the validity and sharpness of Theorem 1.2. In Subsection 3.3, we show the validity of Theorem 1.3 and Theorem 1.4 and characterize the graphs $G$ reaching the lower bound in Theorem 1.4 when $G$ is edge-maximal $K_{p / q}$-colorable. In Subsection 3.5, we show a best possible lower bound for $b(G)$ when $G$ has a graph homomorphism onto an odd cycle $C_{2 p+1}$ and characterize the graphs reaching the lower bound among all edge-maximal $C_{2 p+1}$-colorable graphs. There are a lot of researches about graph homomorphism can be found in [3-7].

\section{An Associate Theorem}

In this section, we shall prove an associate Theorem which will be used in the proofs of other theorems. Throughout this section, we assume that $G$ and $H$ are two graphs and $\varphi$ is an onto graph homomorphism from $G$ to $H$. Note that we can also view $\varphi$ as a map from
$E(G)$ to $E(H)$ such that for each $e=u v \in E(G)$, $\varphi(e)=\varphi(u) \varphi(v) \in E(H)$.

Lemma 2.1 Let $\mathrm{g}$ be a function between sets $X$ and $Y$. Then

1) For any $S \subseteq X, g^{-1}(\bar{S})=\overline{g^{-1}(S)}$.

2) For any sets $C \subseteq Y$ and $D \subseteq Y, C \subseteq D$ if and only if $g^{-1}(C) \subseteq g^{-1}(D)$.

Lemma 2.2 If $D=[S, \bar{S}]$ is an edge cut of $H$, then $\varphi^{-1}(D)=\left[\varphi^{-1}(S), \overline{\varphi^{-1}(S)}\right]$ is an edge cut of $G$. Consequently, $\varphi^{-1}(D)=\left[\varphi^{-1}(S), \varphi^{-1}(\bar{S})\right]$.

Proof. For each $x \in \varphi^{-1}(S), y \in \overline{\varphi^{-1}(S)}$ with $x y \in E(G)$, by the definition of inverse image, $\varphi(x) \in S$, and $\varphi(y) \notin S$. Hence, $\varphi(x y)=\varphi(x) \varphi(y) \in D$, and so $x y \in \varphi^{-1}(D)$. It follows that $\varphi^{-1}(D)=\left[\varphi^{-1}(S), \overline{\varphi^{-1}(S)}\right]$. Conversely, for each $x y \in \varphi^{-1}(D)$, $\varphi(x) \varphi(y)=\varphi(x y) \in D=[S, \bar{S}]$. We may assume that $\varphi(x) \in S$ and $\varphi(y) \in \bar{S}$. Then $x \in \varphi^{-1}(S)$ and $y \notin \varphi^{-1}(S)$. This proves $\varphi^{-1}(D)=\left[\varphi^{-1}(S), \overline{\varphi^{-1}(S)}\right]$. Therefore, $\varphi^{-1}(D)=\left[\varphi^{-1}(S), \overline{\varphi^{-1}(S)}\right]$ is an edge cut of G. By Lemma 2.11$), \varphi^{-1}(D)=\left[\varphi^{-1}(S), \varphi^{-1}(\bar{S})\right]$.

Lemma 2.3 Suppose that $\mathcal{F}=\left\{D_{1}, D_{2}, \cdots, D_{k}\right\}$ is an edge cut cover of $H$. Then

$\mathcal{F}^{\prime}=\left\{\varphi^{-1}\left(D_{1}\right), \varphi^{-1}\left(D_{2}\right), \cdots, \varphi^{-1}\left(D_{k}\right)\right\} \quad$ is an edge cut cover of $G$. Moreover, if an edge $e$ of $H$ lies in exactly $l^{\prime}$ members of $\mathcal{F}$, then every edge in $\varphi^{-1}(e)$ lies in exactly $l^{\prime}$ members of $\mathcal{F}^{\prime}$.

Proof. By the definition of graph homomorphism, for each $e=x y \in E(G), \varphi(x y)=\varphi(x) \varphi(y) \in E(H)$. Since $\mathcal{F}$ is an edge cut cover of $H$, then $\varphi(x y)=\varphi(x) \varphi(y) \in D_{i}$ for some $D_{i} \in \mathcal{F}$. It follows that $x y \in \varphi^{-1}\left(D_{i}\right)$, and so $\mathcal{F}^{\prime}$ is an edge cut cover of $G$. By Lemma 2.1 2), $e \in D_{i}$ if and only if $\varphi^{-1}(e) \subseteq \varphi^{-1}\left(D_{i}\right)$, and so if an edge $e$ of $H$ lies in exactly $l^{\prime}$ members of $\mathcal{F}$, then every edge of $\varphi^{-1}(e)$ lies in exactly $l^{\prime}$ members of $\mathcal{F}^{\prime}$.

Let $k, l$ be two positive integers. A $k$-edge cut at least l-cover of a graph $H$ is a collection $\mathcal{F}=\left\{D_{1}, D_{2}, \cdots, D_{k}\right\}$ of $k$ edge cuts of $H$ such that every edge of $H$ lies in at least $l$ members of $\mathcal{F}$. A $k$-edge cut $l$-cover of a graph $H$ is a collection $\mathcal{F}=\left\{D_{1}, D_{2}, \cdots, D_{k}\right\}$ of $k$ edge cuts of $H$ such that every edge of $H$ lies in exactly $l$ members of $\mathcal{F}$. A $k$-edge cut average $l$-cover of a graph $H$ is a collection $\mathcal{F}=\left\{D_{1}, D_{2}, \cdots, D_{k}\right\}$ of edge cuts of $H$ such that $\bigcup_{i=1}^{k} D_{i}=E(H)$ and $\sum_{i=1}^{k}\left|D_{i}\right|=l|E(H)|$.

Lemma 2.4 Suppose that $\varphi$ is an onto graph homomorphism from $G$ to $H$ and $\mathcal{F}=\left\{D_{1}, D_{2}, \cdots, D_{k}\right\}$ is a $k$ edge cut cover of $H$ such that $\sum_{i=1}^{k}\left|\varphi^{-1}\left(D_{i}\right)\right| \geq l|E(G)|$. 
Then $b(G) \geq \frac{l}{k}|E(G)|$.

Proof. By Lemma 2,

$$
\mathcal{F}^{\prime}=\left\{\varphi^{-1}\left(D_{1}\right), \varphi^{-1}\left(D_{2}\right), \cdots, \varphi^{-1}\left(D_{k}\right)\right\}
$$

is an edge cut cover of $G$ and for any edge $e$ of $H$, if $e$ lies in exactly $l^{\prime}$-members of $\mathcal{F}$, then every edge of $\varphi^{-1}(e)$ lies in exactly $l^{\prime}$ members of $\mathcal{F}^{\prime}$. Therefore, $b(G) \geq \frac{l}{k}|E(G)|$ follows from

$$
l|E(G)| \leq \sum_{i=1}^{k}\left|\varphi^{-1}\left(D_{i}\right)\right| \leq k b(G) .
$$

Theorem 2.5 If one of the following is true, then $b(G) \geq \frac{l}{k}|E(G)|$.

1) There is a graph homomorphism from $G$ onto $H$ and $H$ has a $k$-edge cut $l$-cover.

2) There is a graph homomorphism from $G$ onto $H$ and $H$ has a $k$-edge cut at least $l$-cover.

3) $G$ is edge-uniformly $H$-colorable and $H$ has a $k$-edge cut average $l$-cover.

\section{Main Results}

\subsection{Graphs with a Graph Homomorphism onto a Complete Graph}

In this section, we present an alternative proof for Theorem 1.1 and determine all graphs $G$ reaching the lower bound in Theorem 1.1 when $G$ is edge-maximal $K_{p}$-colorable.

Lemma 3.1 Let $p \geq 2$ be an integer and let $=\left(\begin{array}{c}p \\ \left.\frac{p}{2}\right\rfloor\end{array}\right\rfloor$, $l=\frac{k\left\lfloor\frac{p}{2}\right\rfloor\left\lceil\frac{p}{2}\right\rceil}{\left(\begin{array}{l}p \\ 2\end{array}\right)}$. Then the graph $K_{p}$ has a $k$-edge cut l-cover.

Proof. Let $\mathcal{F}=\left\{\left[X, V\left(K_{p} \backslash X\right)\right]: X\right.$ is a $\left\lfloor\frac{p}{2}\right\rfloor$-subset of $\left.V\left(K_{p}\right)\right\}$. Then $\mathcal{F}$ is an edge cut cover of $K_{p}$ with $|\mathcal{F}|=\left(\begin{array}{c}p \\ \left\lfloor\frac{p}{2}\right.\end{array} \mid\right)=k . \quad$ Since every $\left[X, V\left(K_{p} \backslash X\right)\right] \in \mathcal{F}$ has size $\left.\left|\left[X, V\left(K_{p} \backslash X\right)\right]\right|=\left\lfloor\frac{p}{2}\right\rfloor \mid \frac{p}{2}\right\rceil$, and since
$\left|E\left(K_{p}\right)\right|=\left(\begin{array}{l}p \\ 2\end{array}\right)$, every edge of $K_{p}$ must be in exactly $\frac{k\left\lfloor\frac{p}{2}\right\rfloor\left[\frac{p}{2}\right\rfloor}{\left(\begin{array}{l}p \\ 2\end{array}\right)}=l$ members of $\mathcal{F}$.

Thus, Theorem 1.3 follows from Theorem 2.5 1) and Lemma 3.1.

The lower bound of Theorem 1.1 is best possible, in the sense that there exists a family of graphs such that the lower bound of Theorem 1.1 is reached.

Let $G$ and $H$ be two graphs. The composition of $G$ and $H$, denoted by $G[H]$, is the graph obtained from $G$ by replacing each vertex of $v_{i} \in V(G)$ by $H_{i}$, a copy of $H$, and joining every vertex in $H_{k}$ to every vertex in $H_{l}$ if $v_{k} v_{l} \in E(G)$.

Theorem 3.2 Suppose that there is an onto graph homomorphism from $G$ to $K_{p}$ and $G$ is edge maximal $K_{p}$-colorable.

Then each of the following holds.

1) $b(G) \geq f(p)|E(G)|$, where equality holds only if $G$ is edge-uniformly $K_{p}$-colorable.

2) Among all edge-maximal $K_{p}$-colorable graph $G$, $b(G)=f(p)|E(G)|$ if and only if $G \cong K_{p}\left[\bar{K}_{s}\right]$.

Proof. 1) By Theorem 1.1, $b(G) \geq f(p)|E(G)|$. Suppose that $b(G) \geq f(p)|E(G)|$. Let $\varphi: V(G) \rightarrow V\left(K_{p}\right)$ be an arbitrarily given graph homomorphism and let $V\left(K_{p}\right)=\left\{v_{1}, v_{2}, \cdots, v_{p}\right\}$ be labelled such that if $V_{i}=\varphi^{-1}\left(v_{i}\right)$, then $\left|V_{1}\right| \leq\left|V_{2}\right| \leq \cdots \leq\left|V_{p}\right|$. For any subset $X \subseteq V\left(K_{p}\right)$, let $Y=V\left(K_{p}\right) \backslash X$ and let $\varphi$ also denote the induced map $\varphi: E(G) \rightarrow E\left(K_{p}\right)$. Then $\varphi^{-1}(X)=\bigcup_{v_{i} \in X} \varphi^{-1}\left(v_{i}\right)=\bigcup_{v_{i} \in X} V_{i}$ and $\varphi^{-1}(Y)=V(G) \backslash \varphi^{-1}(X)$. Since $G$ is an edge-maximal $K_{p}$-colorable, $\varphi^{-1}\left([X, Y]_{K_{p}}\right)=\left[\varphi^{-1}(X), \varphi^{-1}(Y)\right]_{V(G)}$ is a complete bipartite graph. There are $\left.k=\left(\begin{array}{c}p \\ \mid \frac{p}{2}\end{array}\right\rfloor\right)$ partitions of $V\left(K_{p}\right)$ into two parts $\left(X_{i}, Y_{i}\right), i=1,2, \cdots, k$, such that $0 \leq\left|Y_{i}\right|-\left|X_{i}\right| \leq 1$. Set $D_{i}=\left[X_{i}, Y_{i}\right]$. Label them so that $\left|\varphi^{-1}\left(D_{1}\right)\right| \leq\left|\varphi^{-1}\left(D_{2}\right)\right| \leq \cdots \leq\left|\varphi^{-1}\left(D_{k}\right)\right|$. By Lemma 2.2 and Lemma 2.3 and the assumption that $b(G)=f(p)|E(G)|, \frac{l}{k}|E(G)|=b(G) \geq\left|\varphi^{-1}\left(D_{k}\right)\right|$ and so $k \cdot \frac{l}{k}|E(G)| \geq k\left|\varphi^{-1}\left(D_{k}\right)\right| \geq \sum_{i=1}^{k}\left|\varphi^{-1}\left(D_{i}\right)\right|=l|E(G)|$, 
where $l=\frac{k\left\lfloor\frac{p}{2}\right\rfloor\left\lceil\frac{p}{2}\right\rfloor}{\left(\begin{array}{c}p \\ 2\end{array}\right)}$. Therefore all the inequalities are equalities and so $\left|\varphi^{-1}\left(D_{1}\right)\right|=\left|\varphi^{-1}\left(D_{2}\right)\right|=\cdots=\left|\varphi^{-1}\left(D_{k}\right)\right|$. Let $X^{\prime}=\left\{v_{1}, v_{i_{1}}, v_{i_{2}}, \cdots, v_{i_{\left\lfloor\frac{p}{2}\right\rfloor}-1}\right\}$ with $v_{p} \notin X^{\prime}, Y^{\prime}=$ $V\left(K_{p}\right) \backslash X^{\prime}$ and $D^{\prime}=\left[X^{\prime}, Y^{\prime}\right]$. Let $X^{\prime \prime}=X^{\prime} \cup\left\{v_{p}\right\} \backslash\left\{v_{1}\right\}$, $Y^{\prime \prime}=V\left(K_{p}\right) \backslash X^{\prime \prime}$ and $D^{\prime \prime}=\left[X^{\prime \prime}, Y^{\prime \prime}\right]$. Then $\left|\varphi^{-1}\left(D^{\prime}\right)\right|=$ $\left|\varphi^{-1}\left(D^{\prime \prime}\right)\right|$. Since

$$
\begin{aligned}
\left|\varphi^{-1}\left(D^{\prime}\right)\right|= & \left|\varphi^{-1}\left(X^{\prime}\right)\right|\left|\varphi^{-1}\left(Y^{\prime}\right)\right| \\
= & \left(\left|V_{1}\right|+\left|V_{i_{1}}\right|+\cdots+\left|V_{i_{\left\lfloor\frac{p}{2}\right.}-1}\right|\right) \\
& +\left(\left|V\left(K_{p}\right)\right|-\left(\left|V_{1}\right|+\left|V_{i_{1}}\right|+\cdots+\left|V_{\left.i^{\prime} \frac{p}{2}\right\rfloor-1}\right|\right)\right)
\end{aligned}
$$

and

$$
\begin{aligned}
\left|\varphi^{-1}\left(D^{\prime \prime}\right)\right| & =\left|\varphi^{-1}\left(X^{\prime \prime}\right)\right|\left|\varphi^{-1}\left(Y^{\prime \prime}\right)\right| \\
& =\left(\left|V_{p}\right|+\left|V_{i_{1}}\right|+\cdots+\left|V_{\left.i_{\mid \frac{p}{2}}\right\rfloor-1}\right|\right) \\
& \left(\left|V\left(K_{p}\right)\right|-\left(\left|V_{p}\right|+\left|V_{i_{1}}\right|+\cdots+\left|V_{i_{\left[\frac{p}{2} \mid-1\right.} \mid}\right|\right)\right),
\end{aligned}
$$

it follows from $\left|\varphi^{-1}\left(D^{\prime}\right)\right|=\left|\varphi^{-1}\left(D^{\prime \prime}\right)\right|$ that $\left|V_{1}\right|=\left|V_{p}\right|$, and so $\left|V_{1}\right|=\left|V_{2}\right|=\cdots=\left|V_{p}\right|=s$ for some positive integer $s$, which implies $G$ is edge uniformly $K_{p}$-colorable.

2) Suppose that $G$ is an edge-maximal $K_{p}$-colorable graph such that $b(G)=f(p)|E(G)|$. By the proof of $1)$, there is some positive integer $s$ such that

$$
G \cong K_{p}\left[\bar{K}_{s}\right]
$$

Conversely, suppose that $G \cong K_{p}\left[\bar{K}_{s}\right]$. By Theorem $1.1, b\left(K_{p}(s)\right) \leq f(p) \mid K_{p}\left[\bar{K}_{s}\right]$. It remains to show that for any partition $(X, Y)$ of $V\left(K_{p}\left[\bar{K}_{s}\right]\right)$,

$$
|[X, Y]| \leq f(p)\left|K_{p}\left[\bar{K}_{s}\right]\right| \text {. }
$$

Let $V_{1}, V_{2}, \cdots, V_{p}$ denote the partition of $V\left(K_{p}\left[\bar{K}_{s}\right]\right)$ such that for $i=1,2, \cdots, p, \quad V_{i}$ is an independent set of $K_{p}\left[\bar{K}_{s}\right]$. Let $[X, Y]$ be an edge cut of $K_{p}(s)$ such that 1) $\mid[X, Y]$ is maximized and subject to 1), 2) $\left|\left\{i:\left|X_{i}\right|\left|Y_{i}\right| \neq 0\right\}\right|$ is minimized, where $X_{i}=X \cap V_{i}$ and $Y_{i}=Y \cap V_{i}$. Then we have the following claims:

Claim 1. For each $i, 1 \leq i \leq p,\left|X_{i}\right|\left|Y_{i}\right|=0$.
Otherwise, there is $i$ such that $\left|X_{i}\right|\left|Y_{i}\right| \neq 0$. Let $m=|X|-\left|X_{i}\right|$ and $t=|Y|-\left|Y_{i}\right|$, If $m=t$, let $X^{\prime}=X \cup Y_{i}$ and $Y^{\prime}=Y \backslash Y_{i}$ Then $\left[X^{\prime}, Y^{\prime}\right]$ is an edge cut of $K_{p}\left[\bar{K}_{s}\right]$ such that $|[X, Y]|=\mid\left[X^{\prime}, Y^{\prime}\right]$ and $\left|\left\{i:\left|X_{i}^{\prime}\right|\left|Y_{i}^{\prime}\right| \neq 0\right\}\right|=\left|\left\{i:\left|X_{i}\right|\left|Y_{i}\right| \neq 0\right\}\right|-1$, contrary to the choice of $[X, Y]$. Then $m \neq t$. Without less of generality, we may assume $m<t$. Let $X^{\prime}=X \cup Y_{i}$ and $Y^{\prime}=Y \backslash Y_{i}$. Then $\left[X^{\prime \prime}, Y^{\prime \prime}\right]$ is an edge cut of $K_{p}\left[\bar{K}_{s}\right]$ such that $\left|\left[X^{\prime \prime}, Y^{\prime \prime}\right]\right|=|[X, Y]|+(t-m)\left|Y_{i}\right|>|[X, Y]|$, contrary to the choice of $[X, Y]$.

Claim 2. $\mid\left\{\left\{i: V_{i} \subseteq X\right\}|-|\left\{i: V_{i} \subseteq Y\right\} \| \leq 1\right.$.

Otherwise, ||$\left\{i: V_{i} \subseteq X\right\}|-|\left\{i: V_{i} \subseteq Y\right\} \mid \geq 2$. Choose $V_{i} \subseteq X$ and set $X^{\prime}=X \backslash V_{i}$ and $Y^{\prime}=Y \bigcup V_{i}$. Then $\left[X^{\prime}, Y^{\prime}\right]$ is an edge cut such that

$$
\begin{aligned}
\left|\left[X^{\prime}, Y^{\prime}\right]\right| & =|[X, Y]|+\left(\left|\left\{i: V_{i} \subseteq X\right\}\right|-\left|\left\{i: V_{i} \subseteq Y\right\}\right|-1\right) m^{2} \\
& >|[X, Y]|,
\end{aligned}
$$

contrary to the choice of $[X, Y]$.

By Claim 1 and Claim 2, we have $|[X, Y]|=\frac{p^{2} s^{2}}{4}$ when $p$ is even and $|[X, Y]|=\frac{\left(p^{2}-1\right) s^{2}}{4}$ when $p$ is odd, that is, $|[X, Y]|=f(p) \mid E\left(K_{p}\left[\bar{K}_{s}\right]\right)$.

\subsection{Graphs with a Graph Homomorphism onto a Kneser Graph}

In this section, we shall show the validity and sharpness of Theorem 1.2.

Theorem 3.3 Let $p$ and $q$ be two positive integers with $p \geq 2 q$. Then $K_{p: q}$ has a $p$-edge cut $2 q$-cover.

Proof. For $i=0,1, \cdots, p-1$, let $V_{i}=\{X \subseteq\{0,1,2, \cdots, p-1\}:|X|=q$ and $i \in X\}$ and $D_{i}=\left[V_{i}, \bar{V}_{l}\right]$. Then $\left|V_{i}\right|=\left(\begin{array}{c}p-1 \\ q-1\end{array}\right)$. By the definition of $K_{p: q}, V_{i}$ is an independent sets of $K_{p: q}$ and $D_{i}$ is an edge cut of size $\left(\begin{array}{l}p-1 \\ q-1\end{array}\right) \times\left(\begin{array}{c}p-q \\ q\end{array}\right)$. Then $\mathcal{F}=\left\{D_{0}, D_{1}, \cdots, D_{p-1}\right\}$ is an edge cut cover of $K_{p: q}$. Since $K_{p: q}$ has $\left(\begin{array}{l}p \\ q\end{array}\right)$ vertices and each of them is of degree $\left(\begin{array}{c}p-q \\ q\end{array}\right),\left|E\left(K_{p: q}\right)\right|=\frac{\left(\begin{array}{l}p \\ q\end{array}\right)\left(\begin{array}{c}p-q \\ q\end{array}\right)}{2}$.

Since each edge of $K_{p: q}$ is contained in 
$\frac{\left(\begin{array}{c}p-1 \\ q-1\end{array}\right) \times\left(\begin{array}{c}p-q \\ q\end{array}\right) \times p}{\left(\begin{array}{l}p \\ q\end{array}\right) \times\left(\begin{array}{c}p-q \\ q\end{array}\right)}=2 q$ edges cuts, $K_{p: q}$ has a $p$-edge

cut $2 q$-cover.

Proof of Theorem 1.2 Theorem 1.2 follows from Theorem 2.5 and Theorem 3.3.

Theorem 3.4 (Poljak and Tuza, Theorem 2 in [8]). If $p \leq 3 q$, then the maximum edge cut of $K_{p: q}$ is induced by the maximum independent set of $K_{p: q}$ and is of size $\left(\begin{array}{c}p-1 \\ q-1\end{array}\right) \times\left(\begin{array}{c}p-q \\ q\end{array}\right)$

Theorem 3.5 The bound in Theorem 1.2 is best possible.

Proof. By Theorem 3.4, the edge cuts we choose in the proof of Theorem 4.1 are the maximum edge cuts of $K_{p: q}$ when $p \leq 3 q$. Therefore, the bound in Theorem 1.2 is reached by $K_{p: q}$ when $p \leq 3 q$.

\subsection{Graphs with a Graph Homomorphism onto a Circulant Graph}

In this section, we verify the validity and sharpness of Theorem 1.3 and Theorem 1.4.

Theorem 3.6 Let $p$ and $q$ be two positive integers with $p \geq 2 q$. Then the following hold.

1) $K_{p / q}$ has a $p$-edge cut at least $2 q$-cover.

2) $K_{p / q}$ has a $p$-edge cut average

$\frac{2\left(\left\lfloor\frac{p}{2}\right\rfloor\left\lceil\frac{p}{2}\right\rceil-q(q-1)\right)}{p-1-2(q-1)}$-cover.

Proof. For $i=0,1, \cdots, p-1$, let

$V_{i}=\left\{i, i+1, \cdots,\left\lfloor\frac{p}{2}\right\rfloor-1\right\}$ and let $D_{i}=\left[V_{i}, \bar{V}_{i}\right]$. Then $\mathcal{F}=\left\{D_{0}, D_{1}, \cdots, D_{p-1}\right\}$ is an edge cut cover of $K_{p / q}$ with $\left|D_{i}\right|=\left\lfloor\frac{p}{2}\right\rfloor\left\lceil\frac{p}{2}\right\rceil-q(q-1)$.

1) Notice that for any edge $e=u v \in K_{p / q}, e$ lies in at least $2 q$ members of $\mathcal{F}=\left\{D_{0}, D_{1}, \cdots, D_{p-1}\right\}$, then $K_{p / q}$ has a $p$-edge cut at least $2 q$-cover.

$$
\begin{aligned}
& \text { 2) Since }\left|E\left(K_{\frac{p}{q}}\right)\right|=\frac{p(p-1-2(q-1))}{2} \text {, } \\
& \sum_{i=0}^{p}\left|D_{i}\right|=\left(\left\lfloor\frac{p}{2} \mid\left\lceil\frac{p}{2}\right\rceil-q(q-1)\right) p\right. \\
& =\frac{2\left(\left\lfloor\frac{p}{2}\right\rfloor\left\lceil\frac{p}{2}\right\rceil-q(q-1)\right)}{p-1-2(q-1)}\left|E\left(K_{p / q}\right)\right|
\end{aligned}
$$

and so $K_{p / q}$ has a $p$-edge cut average

$$
\frac{2\left(\left\lfloor\frac{p}{2}\right\rfloor\left\lceil\frac{p}{2}\right\rceil-q(q-1)\right)}{p-1-2(q-1)} \text {-cover. }
$$

Proof of Theorem 1.3 Theorem 1.3 follows from Theorem 2.5 and Theorem 3.61 ).

Proof of Theorem 1.4 Theorem 1.4 follows from Theorem 2.5 and Theorem 3.62 ).

Theorem 3.7 Let $G$ be an edge-maximal graph such that $G$ is edge uniformly $K_{p / q}$-colorable. Then $G \cong K_{p / q}\left[\bar{K}_{s}\right]$ and $b(G)=f(p, q)|E(G)|$.

Proof. Suppose that $G$ is an edge-maximal graph such that $G$ is edge-uniformly $K_{p / q}$-colorable. Then, $G \cong K_{p / q}\left[\bar{K}_{s}\right]$ for some positive integer s. By Theorem 1.4, we have $b\left(K_{p / q}\left[\bar{K}_{s}\right]\right) \geq f(p, q)\left|E\left(K_{p / q}\left[\bar{K}_{s}\right]\right)\right|$.

Now we prove that $b\left(K_{p / q}\left[\bar{K}_{s}\right]\right)=f(p, q)\left|E\left(K_{p / q}\left[\bar{K}_{s}\right]\right)\right|$.

For $i \in\{0,1, \cdots, p-1\}=V\left(K_{p / q}\right)$, let $V_{i}=h^{-1}(i)$, where $h: V(G) \rightarrow V\left(K_{p / q}\right)$ is an onto graph homomorphism from $G$ to $K_{p / q}$ such that for any edge $x y$ of $G$, $q \leq|h(x)-h(y)| \leq p-q$. By the definition of graph homomorphism, $V_{i}$ is an independent set. Let $[X, Y]$ be an edge cut of $K_{p / q}\left[\bar{K}_{s}\right]$ such that 1$) \mid[X, Y] \|$ is maximized and subject to 1), 2) $\quad\left\{i:\left|X_{i}\right|\left|Y_{i}\right| \neq 0\right\} \mid$ is minimized, where $X_{i}=X \cap V_{i}$ and $Y_{i}=Y \cap V_{i}$.

Claim 1. For each $i,\left|X_{i}\right|\left|Y_{i}\right|=0$.

Proof. Otherwise, there is $i$ such that $\left|X_{i}\right|\left|Y_{i}\right| \neq 0$. Since for each $v_{i} \in V_{i}$, either $\left|N\left(v_{i}\right) \cap X\right| \geq\left|N\left(v_{i}\right) \cap Y\right|$ or $\left|N\left(v_{i}\right) \cap X\right| \leq\left|N\left(v_{i}\right) \cap Y\right|$. If the former is true, then $\left|\left[X \backslash X_{i}, Y \cup X_{i}\right]\right| \geq|[X, Y]|$, contradicting the choice of $[X, Y]$; if the latter is true, then $\left|\left[X \cup Y_{i}, Y \backslash Y_{i}\right]\right| \geq|[X, Y]|$, contradicting the choice of $[X, Y]$.

Assume that $X=\bigcup_{i=j_{1}}^{j_{m}} V_{i}$ and let $\left\{j_{1}, j_{2}, \cdots, j_{m}\right\}=J$. Without loss of generality, we can assume that $|J| \leq\left\lfloor\frac{p}{2}\right\rfloor$. Let $C_{p}$ be the cycle with vertex set $\{0,1,2, \cdots, p-1\}=V\left(K_{p / q}\right)$, where $i$ is adjacent to $j$ if and only if $|i-j|=1(\bmod p)$. Let $\operatorname{dist}_{C_{p}}(J)$ be the length of the shortest path of $C_{p}$ which contains all the elements in $J$. Then $\operatorname{dist}_{C}(J) \geq m-1$.

Claim 1. $\operatorname{dist}_{C}(J)=m-1$.

Proof. Suppose, to the contrary, that $\operatorname{dist}_{C_{p}}(J)>m-1$. Let $P$ be a path of $C_{p}$ which contains all the elements 
in $J$ and assume that $j_{1}, j_{m}$ are the endpoints of $P$. Then there is $k \in V(P) \backslash J$. Let $X^{\prime}=\left(X \backslash\left\{V_{j_{m}}\right\}\right) \cup\left\{V_{m}\right\}$ and $J^{\prime}=J \backslash\left\{j_{m}\right\} \cup\{m\}$. Then $\operatorname{dist}_{C_{p}}\left(J^{\prime}\right)<\operatorname{dist}_{C_{p}}(J)$ and $\left|\left[X^{\prime}, \bar{X}^{\prime}\right]\right|>|[X, \bar{X}]|$, a contradiction.

Claim 2. $m=\left\lfloor\frac{p}{2}\right\rfloor$.

Proof. Suppose, to the contrary, that $m<\left\lfloor\frac{p}{2}\right\rfloor$. Let $P$ be a path of $C_{p}$ which contains all the elements in $J$ and assume that $j_{1}, j_{m}$ are the endpoints of $P$. Let $j_{m+1} \in V\left(C_{p}\right) \backslash V(P)$ be such that $j_{m} j_{m+1} \in E\left(C_{p}\right)$. Let $X^{\prime}=X \bigcup\left\{V_{m+1}\right\}$ and $J^{\prime}=J \bigcup\left\{j_{m+1}\right\}$. Then

$\operatorname{dist}_{C}\left(J^{\prime}\right)=\operatorname{dist}_{C}(J)+1 \leq\left\lfloor\frac{p}{2}\right\rfloor$ and $\left|\left[X^{\prime}, \bar{X}^{\prime}\right]\right|>|[X, \bar{X}]|$, a contradiction.

By the above discussion, we can calculate that $b\left(K_{p / q}\left[\bar{K}_{s}\right]\right)=f(p, q)\left|E\left(K_{p / q}(s)\left[\bar{K}_{s}\right]\right)\right|$.

\subsection{Graphs with a Graph Homomorphism onto an Odd Cycle}

In this section, we will show a best possible lower bound for $b(G)$ when $G$ has a graph homomorphism onto an odd cycle $C_{2 p+1}$ and characterize the graphs reaching the lower bound among all edge-maximal

$C_{2 p+1}$-colorable graphs.

Theorem 3.8 Suppose that there is an onto graph homomorphism from $G$ to $C_{2 p+1}$. Then each of the following holds.

1) $b(G) \geq \frac{2 p}{2 p+1}|E(G)|$, where equality holds only if $G$ is edge-uniformly $C_{2 p+1}$-colorable.

2) Among all edge-maximal $C_{2 p+1}$-colorable graph $G$, $b(G)=\frac{2 p}{2 p+1}|E(G)|$ if and only if $G \cong C_{2 p+1}\left[\bar{K}_{s}\right]$.

Proof. 1) Notice that $C_{2 p+1} \cong K_{(2 p+1) /(2 p)}$, $b(G) \geq \frac{2 p}{2 p+1}|E(G)|$ follows from Theorem 1.3. Now suppose that $b(G)=\frac{2 p}{2 p+1}|E(G)|$. Let

$\varphi: V(G) \rightarrow V\left(C_{2 p+1}\right)$ be an arbitrarily given graph homomorphism and for each $i \in Z_{2 p+1}$, let $V_{i}=\varphi^{-1}\left(v_{i}\right)$. Set $W_{i}=\left[V_{i}, V_{i+1}\right]$, for each $i \in Z_{2 p+1}$. Since $\varphi$ is a graph homomorphism, we have $E(G)=\bigcup_{i \in Z_{2 p+1}} W_{i}$. It follows that $|E(G)|=\sum_{i=0}^{2 p}\left|W_{i}\right|$. We may assume that $\left|W_{0}\right|=\min \left\{\left|W_{i}\right|: i \in Z_{2 p+1}\right\}$. Then $E(G)-W_{0}$ is an edge- cut of $G$, and so by the assumption on $G$, we have $\frac{2 p}{2 p+1}|E(G)|=b(G) \geq|E(G)|-\left|W_{0}\right|=\sum_{i=1}^{2 p}\left|W_{i}\right|$. It follows that $2 p\left(\sum_{i=0}^{2 p}\left|W_{i}\right|\right)=2 p|E(G)| \geq(2 p+1) \sum_{i=1}^{2 p}\left|W_{i}\right|$. Hence $2 p\left|W_{0}\right| \geq\left|W_{1}\right|+\left|W_{1}\right|+\cdots+\left|W_{2 p}\right|$. By the choice of $X_{0}$, we must have $\left|W_{0}\right|=\left|W_{1}\right|=\cdots=\left|W_{2 p}\right|$. Let $m=\left|W_{0}\right|$. Then for any edge $e \in E\left(C_{2 p+1}\right),\left|\varphi^{-1}(e)\right|=m$. Since $\varphi$ is arbitrarily, then $G$ is edge-uniformly $C_{2 p+1}$-colorable.

2) Suppose that $G$ is an edge-maximal $C_{2 p+1}$-colorable graph with $b(G)=\frac{2 p}{2 p+1}|E(G)|$. Let $W_{i}$ be defined as in 1). Since $G$ is an edge-maximal $C_{2 p+1}$-colorable graph, the subgraph induces by $W_{i}$ is a complete bipartite graph. Since

$b(G)=\frac{2 p}{2 p+1}|E(G)|$, by 1$), G$ is edge-uniformly $C_{2 p+1}$ colorable, which means, $\left|W_{0}\right|=\left|W_{1}\right|=\cdots=\left|W_{2 p}\right|$ Therefore, $\left|V_{0}\right|=\left|V_{1}\right|=\cdots=\left|V_{2 p}\right|=s$ for some positive integer $s$ and so $G \cong C_{2 p+1}\left[\bar{K}_{s}\right]$.

Conversely, suppose $G \cong C_{2 p+1}\left[\bar{K}_{s}\right]$. Note that $\left|E\left(C_{2 p+1}\left[\bar{K}_{s}\right]\right)\right|=(2 p+1) \mathrm{m}^{2}$. Then $b\left(C_{2 p+1}\left[\bar{K}_{s}\right]\right) \geq 2 \mathrm{pm}^{2}$ and so it suffices to show that for any subset $\varnothing \neq X \subseteq V\left(C_{2 p+1}\left[\bar{K}_{s}\right]\right)$ and $Y=V\left(C_{2 p+1}\left[\bar{K}_{s}\right]\right) \backslash X$, the edge cut $[X, Y]$ of $C_{2 p+1}\left[\bar{K}_{s}\right]$ satisfies $|[X, Y]| \leq 2 \mathrm{pm}^{2}$.

Let $[X, Y]$ be an edge cut of $C_{2 p+1}\left[\bar{K}_{s}\right]$ such that 1) $|[X, Y]|$ is maximized and subject to 1$), 2$ ) $\left|\left\{i:\left|X_{i}\right|\left|Y_{i}\right| \neq 0\right\}\right|$ is minimized, where $X_{i}=X \cap V_{i}$ and $Y_{i}=Y \cap V_{i}$. Since $\left[\bigcup_{i \in I_{1}} V_{i}, \bigcup_{i \in I_{2}} V_{i}\right]$, where $I_{1}=\left\{i \in Z_{2 p+1}: i\right.$ is odd $\}$ and $I_{2}=\left\{i \in Z_{2 p+1}: i\right.$ is even $\}$, is an edge cut with cardinality $2 \mathrm{pm}^{2}$, then

$|[X, Y]| \geq\left|\left[\bigcup_{i \in I_{1}} V_{i}, \bigcup_{i \in I_{2}} V_{i}\right]\right| \geq 2 \mathrm{pm}^{2}$. Then we have the following claims:

Claim 1. For each $i,\left|X_{i}\right|\left|Y_{i}\right|=0$.

Proof. Otherwise, there is $i$ such that $\left|X_{i}\right|\left|Y_{i}\right| \neq 0$. Let $s=\left|X_{i+1}\right|+\left|X_{i-1}\right|$ and $t=\left|Y_{i+1}\right|+\left|Y_{i-1}\right|$. If $s=t$, let $X^{\prime}=X \cup Y_{i}$ and $Y^{\prime}=Y \backslash Y_{i}$. Then $\left[X^{\prime}, Y^{\prime}\right]$ is an edge cut of $G_{m}$ such that $|[X, Y]|=\left|\left[X^{\prime}, Y^{\prime}\right]\right|$ and 
$\$\left|\left\{i:\left|X_{i}^{\prime}\right|\left|Y_{i}^{\prime}\right| \neq 0\right\}\right|=\mid\left\{i:\left|X_{i}^{\prime}\right|\left|Y^{\prime} \neq 0_{i}\right|-1\right.$, contrary to the choice of $[X, Y]$. Then $s \neq t$. Without loss of generality, we may assume $s<t$. Let $X^{\prime \prime}=X \cup Y_{i}$ and $Y^{\prime \prime}=Y \backslash Y$. Then $\left[X^{\prime \prime}, Y^{\prime \prime}\right]$ is an edge cut of $G_{m}$ such that $\left|\left[X^{\prime \prime}, Y^{\prime \prime}\right]\right|=|[X, Y]|+(t-s)\left|Y_{i}\right|>|[X, Y]|$, contrary to the choice of $[X, Y]$.

Claim 2. There is exactly one pair of consecutive number $\{i, i+1\}$ in $\{0,1, \cdots, 2 p\}$ (we consider 0 and $2 p$ as a pair of consecutive numbers, too) such that $\left|X_{i}\right|=\left|X_{i+1}\right|$.

Proof. By Claim 1 and the structure of $C_{2 p+1}\left[\bar{K}_{s}\right]$, there exist some pair of consecutive numbers $\{i, i+1\}$ in $\{0,1, \cdots, 2 p\}$ such that $\left|X_{i}\right|=\left|X_{i+1}\right|$ Since $|[X, Y]| \geq 2 \mathrm{pm}^{2}$, then there is at most one pair of consecutive number $\{i, i+1\}$ in $\{0,1, \cdots, 2 p\}$ such that $\left|X_{i}\right|=\left|X_{i+1}\right|$ and so Claim 2 follows.

By Claim 1 and Claim 2, $|[X, Y]| \geq 2 \mathrm{pm}^{2}$.

\section{References}

[1] A. J. Bondy and U. S. R. Murty, "Graph Theory with
Applications,” American Elsevier, New York, 1976.

[2] P. Erdös, "Problems and Results in Graph Theory and Combinatorial Analysis," Graph Theory and RelatedTopics, Academic Press, New York, 1979.

[3] M. O. Albertson and K. L. Gibbons, "Homomorphisms of 3-Chromatic Graphs," Discrete Mathematics, Vol. 54, No. 2, 1985, pp. 127-132. doi:10.1016/0012-365X(85)90073-1

[4] M. O. Albertson, P. A. Catlin and L. Gibbons, "Homomorphisms of 3-Chromatic Graphs II," Congressus Numerantium, Vol. 47, 1985, pp. 19-28.

[5] P. A. Catlin, "Graph Homomorphisms onto the 5-Cycle," Journal of Combinatorial Theory, Series B, Vol. 45, No. 2, 1988, pp. 199-211. doi:10.1016/0095-8956(88)90069-X

[6] E. R. Scheinerman and D. H. Ullman, "Fractional Graph Theory,” John Wiley Sons, Inc., New York, 1997.

[7] G. G. Gao and X. X. Zhu, "Star-Extremal Graphs and the Lexicographic Product," Discrete Mathematics, Vol. 153, 1996, pp. 147-156.

[8] S. Poljak and Z. Tuza, "Maximum Bipartite Subgraphs of Kneser Graphs," Graphs and Combinatorics, Vol. 3, 1987, pp. 191-199. doi:10.1007/BF01788540 